\title{
The bidirectional Glenn operation: A risk factor analysis for morbidity and mortality
}

\author{
Brian E. Kogon, MD, ${ }^{\text {a }}$ Courtney Plattner, BA, ${ }^{a}$ Traci Leong, PhD, ${ }^{\mathrm{b}}$ Janet Simsic, MD, ${ }^{\mathrm{c}}$ Paul M. Kirshbom, MD, ${ }^{\mathrm{a}}$ and \\ Kirk R. Kanter, $\mathrm{MD}^{\mathrm{a}}$
}

Earn CME credits at http://

cme.ctsnetjournals.org
From the Division of Cardiothoracic Surgery, Emory University School of Medicine, ${ }^{\text {a }}$ Atlanta, Ga; Rollins School of Public Health, ${ }^{\mathrm{b}}$ Atlanta, Ga; and Sibley Cardiology, Children's Healthcare of Atlanta, ${ }^{\mathrm{c}}$ Atlanta, Ga.

Received for publication Dec 11, 2007; revisions received March 25, 2008; accepted for publication May 4, 2008.

Address for reprints: Brian E. Kogon, MD, Emory University, Children's Healthcare of Atlanta, Egleston, Atlanta, GA (E-mail: Brian_kogon@emoryhealthcare.org).

J Thorac Cardiovasc Surg 2008;136:1237 42

\section{$0022-5223 / \$ 34.00$}

Copyright $\odot 2008$ by The American Association for Thoracic Surgery

doi:10.1016/j.jtcvs.2008.05.017
Objective: Patients with single ventricle heart defects often undergo a palliative bidirectional Glenn operation. For this operation, we analyzed potential risk factors for morbidity and mortality. We also evaluated the effects of a persistent left superior vena cava by comparing the outcomes of unilateral and bilateral operations.

Methods: We reviewed the clinical records of 270 consecutive patients who underwent a bidirectional Glenn operation between 2001 and 2007. A total of 226 patients underwent unilateral operations and 44 patients underwent bilateral operations. Patient characteristics included weight and age, single ventricle morphology, vena caval anatomy, and previous surgery. Operative details included cardiopulmonary bypass technique and duration, pulmonary artery management, hemi-Fontan construction, concomitant procedures, and hemodynamics. Outcome data included duration of chest tube drainage, lengths of intensive care unit and hospital stay, morbidity, and mortality ( $<30$ days).

Results: The median length of chest tube drainage was 2.4 days (range 1-20 days). Risk factors for prolonged drainage were elevated central venous pressure $(P=.015)$ and transpulmonary gradient $(P=.011)$. The median lengths of stay in the intensive care unit and hospital were 50 hours (range 20-1628 hours) and 5 days (range 2-83 days), respectively. Risk factors for both included prolonged cardiopulmonary bypass time, elevated central venous pressure and transpulmonary gradient, and right ventricular morphology. Overall, 72 of 270 patients (27\%) had 116 postoperative complications. Risk factors included prolonged cardiopulmonary bypass time $(P=.002)$ and elevated central venous pressure $(P=.029)$. Mortality was 2 of 270 patients $(0.7 \%)$. No risk factors for death were identified. Weight (median $6.8 \mathrm{~kg}$ vs $6.2 \mathrm{~kg}$, $P=.038$ ) and age (median 186 days vs 159 days, $P=.001$ ) at the time of surgery were significantly greater in the bilateral bidirectional Glenn group compared with the unilateral group. However, there was no difference in any of the outcome variables.

Conclusion: Outcomes were adversely affected primarily by prolonged cardiopulmonary bypass time, elevated central venous pressure and transpulmonary gradient, and right ventricular morphology. Specifically, outcomes were unaffected by the presence of a left superior vena cava, cannulation strategy, or antegrade pulmonary blood flow. There were few differences between the unilateral and bilateral groups, none of which were postoperative outcomes.

$\mathrm{P}$ atients with congenital heart defects requiring single ventricle palliation typically undergo a bidirectional Glenn procedure. For this operation, the optimal timing of surgery, technique of cardiopulmonary bypass (CPB) and cannulation, and main pulmonary management are sometimes unclear. In hopes of resolving some of these issues, the potential risk factors for morbidity and mortality were analyzed, along with the effects of a persistent left superior vena cava.

\section{Materials and Methods}

After obtaining internal review board approval, we retrospectively reviewed the clinical and surgical records of 270 pediatric patients who underwent a bidirectional Glenn procedure between 2001 and 2007 at a single institution. We reviewed preoperative, operative, and 


\section{Abbreviation and Acronym}

$\mathrm{CPB}=$ cardiopulmonary bypass

postoperative data. Comparisons were made between bilateral and unilateral operations, and a risk factor analysis was performed for morbidity and mortality.

\section{Patients}

Overall, the median weight and age at the time of surgery was $6.3 \mathrm{~kg}$ (range, 4.2-25.1 kg) and 164 days (range, 76-4155 days). There were 13 patients aged between 2 and 3 months, 36 patients aged between 3 and 4 months, 61 patients aged between 4 and 5 months, and 54 patients aged between 5 and 6 months (Table 1). The remaining 106 patients were aged more than 6 months of age. Anatomically, 132 patients (49\%) had a morphologic single left ventricle, 120 patients $(44 \%)$ had a morphologic single right ventricle, and 18 patients $(7 \%)$ had either 2 ventricles that could not be partitioned or indeterminate single ventricular morphology. In addition, 12 patients (4\%) had associated interrupted inferior vena cava with azygous or hemizygous continuation to a superior vena cava. A total of 292 operations were performed before the bidirectional Glenn operaton in 222 of 270 patients $(82 \%)$.

\section{Operation}

With one exception, all of the operations were performed using CPB, with a mean CPB time of $74 \pm 34$ minutes (Table 2). For the 91 patients (34\%) who underwent a period of cardioplegic arrest, the mean ischemic time was $28 \pm 18$ minutes. For the 28 patients $(10 \%)$ who underwent a period of fibrillatory arrest, the mean fibrillation time was $10 \pm 10$ minutes. In most cases, fibrillatory arrest was used for ligation of the main pulmonary artery and oversewing of the pulmonary valve, atrial septectomy, or atrioventricular valvuloplasty. In the bilateral bidirectional group, bilateral superior vena caval cannulation was achieved in 24 of 44 patients (55\%). Modified ultrafiltration was used in all patients who weighed less than $10 \mathrm{~kg}$. At the conclusion of the operation, antegrade pulmonary blood flow was absent in 218 of 270 patients $(81 \%)$ because of anatomic pulmonary atresia, prior Norwood operation, prior or concomitant DamusKaye-Stansel anastomosis, or concomitant main pulmonary artery ligation. The remaining 52 of 270 patients (19\%) had some degree of antegrade pulmonary blood flow limited by native pulmonary stenosis or restricted by banding of the pulmonary artery. By excluding the takedown of a right ventricle-to-pulmonary artery or Blalock-Taussig shunt, 252 concomitant procedures were performed in 165 of 270 patients (61\%). After weaning from CPB, mean central venous pressure was $13.6 \pm 2.6 \mathrm{~mm} \mathrm{Hg}$ and common atrial pressure was $5.8 \pm 2.1 \mathrm{~mm} \mathrm{Hg}$, resulting in a transpulmonary gradient of $7.8 \pm 2.1 \mathrm{~mm} \mathrm{Hg}$.

\section{Outcome Data}

Postoperative outcome data included duration of chest tube drainage, lengths of intensive care unit and hospital stay, morbidity, and mortality ( $<30$ days). The duration of chest tube drainage was determined by the day on which all of the intraoperative chest tubes were removed. Morbidity was determined by the presence
TABLE 1. Summary of patient characteristics

\begin{tabular}{lcc}
\hline & $\begin{array}{c}\text { No. of patients } \\
(\%)(\mathbf{n}=270)\end{array}$ & $\begin{array}{c}\text { Median } \\
\text { (range) }\end{array}$ \\
\hline $\begin{array}{l}\text { Preoperative factors } \\
\text { Weight at surgery (kg) }\end{array}$ & \\
Age at surgery (d) & & $164(76-2-25.1)$ \\
Single ventricle morphology & & \\
$\quad$ Left & $132(49 \%)$ & \\
$\quad$ Right & $120(44 \%)$ & \\
$\quad$ Other & $18(7 \%)$ & \\
Presence of bilateral SVC & $44(16 \%)$ & \\
Presence of an interrupted IVC & $12(4 \%)$ & \\
Previous operations & $222(82 \%)$ &
\end{tabular}

SVC, Superior vena cava; $I V C$, inferior vena cava.

of a complication. Complications were grouped into categories: dysrhythmia (28), effusions (22), respiratory (18), reoperation (17), infection (11), open chest (6), cardiac failure (5), neurologic (4), and other (5). Specifically, effusive complications were considered present if a pericardial or pleural effusion required placement of an additional chest tube in the postoperative period, or if a chylous effusion required dietary changes to a no-fat or low-fat diet.

\section{Statistics}

In comparing the unilateral and bilateral populations, statistical analysis was performed using the Mann-Whitney test for continuous variables and chi-square test for categoric variables. In evaluating potential risk factors for outcomes, a regression model was used. A multiple regression analysis was performed for outcomes with $P$ values less than .1.

\section{TABLE 2. Summary of operative details}

No. of patients

$(\%)(n=270) \quad$ Mean \pm SD

Operative factors
Use of CPB
CPB time (min) $(\mathrm{n}=269)$
Use of cardioplegic arrest
Ischemic time (min) $(\mathrm{n}=91)$
Use of fibrillatory arrest
Fibrillation time (min) ( $\mathrm{n}=28)$
Maintenance of antegrade PA flow
Hemi-Fontan construction
Concomitant procedures
(except shunt/conduit takedown)
Hemodynamics
CVP (mm Hg)
TP gradient (mm Hg)
CA pressure $(\mathrm{mm} \mathrm{Hg})$

$269(100 \%)$

$91(34 \%)$

$28(10 \%)$

$52(19 \%)$

$14(5 \%)$

$165(61 \%)$

$74 \pm 34$

$28 \pm 18$

$10 \pm 10$

$S D$, Standard deviation; $C P B$, cardiopulmonary bypass; $P A$, pulmonary artery; $C V P$, central venous pressure; $T P$, transpulmonary; $C A$, common atrial. 
TABLE 3. Summary of postoperative outcomes

\begin{tabular}{lccl}
\hline & $\begin{array}{c}\text { No. of patients } \\
(\%)(\mathbf{n}=270)\end{array}$ & Median & (Range) \\
\hline Outcomes & & & \\
Chest tube drainage (d) & & 2.4 & $(1-20)$ \\
ICU duration (h) & & 50 & $(20-1628)$ \\
LOS (d) & & 5 & $(2-83)$ \\
Complications & $72(27 \%)$ & & \\
Surgical mortality $(<30 \mathrm{~d})$ & $2(0.7 \%)$ & & \\
\hline
\end{tabular}

$I C U$, Intensive care unit; $L O S$, length of stay.

\section{Results}

Outcome Summary

The median length of chest tube drainage was 2.4 days (range 1-20 days) (Table 3). The median lengths of stay in the intensive care unit and hospital were 50 hours (range 20-1628 hours) and 5 days (range 2-83 days), respectively. Overall,

\section{TABLE 4. Complications}

Rhythm complications

AV block requiring temporary pacemaker

Arrhythmia

Effusive complications

Pericardial effusion requiring re-drainage

Pleural effusion requiring re-drainage

Chylothorax requiring no-fat or low-fat diet

Respiratory complications

Pneumothorax

Phrenic nerve injury/diaphragm paralysis

Pulmonary hypertensive crisis

Respiratory insufficiency requiring reintubation

Mechanical ventilation $>7 \mathrm{~d}$

Reoperative complications

Unplanned reoperation

Bleeding requiring reoperation

Infectious complications

Pneumonia

Wound infection

Mediastinitis

Septicemia

Open sternum

Open sternum

Cardiac complications

Low cardiac output

Cardiac arrest

Mechanical circulatory support

Neurologic complications

Neurologic deficit persisting at discharge

New-onset seizures

Other complications

Other
72 of 270 patients (27\%) had 116 postoperative complications (Table 4). Surgical mortality was 2 of 270 patients $(0.7 \%)$.

\section{Risk Factor for a Prolonged Intensive Care Unit Stay and Hospital Stay}

A risk factor analysis for prolonged intensive care unit and hospital stay was performed (Table 5). Longer CPB time, elevated central venous pressure and transpulmonary gradient, and right ventricular morphology were risk factors for both. In addition, a lower weight at the time of surgery affected the length of hospital stay $(P=.031)$. Only right ventricular morphology maintained significance in the multivariate analysis.

\section{Risk Factors for Overall Morbidity and Mortality}

A risk factor analysis for overall complications and death was performed (Table 6). Risk factors for any complication included longer CPB time $(P=.002)$ and elevated central venous pressure $(P=.029)$. Only prolonged CPB maintained significance in the multivariate analysis. With such low mortality $(0.7 \%)$, no risk factors for death were identified.

\section{Risk Factors for Specific Complications}

By looking specifically at effusions, the presence of residual antegrade pulmonary artery flow had no effect on the duration of operative chest tube drainage $(P=.50)$ or the presence of subsequent effusive complications $(P=.42)$ (Tables 5 and 7). On the other hand, the duration of initial

TABLE 5. Summary of univariate risk analysis for chest tube drainage, intensive care unit stay, and hospital stay

\begin{tabular}{|c|c|c|c|}
\hline & $\begin{array}{c}\text { CT } \\
\text { drainage (d) }\end{array}$ & $\begin{array}{l}\text { ICU } \\
\text { (h) }\end{array}$ & $\begin{array}{l}\text { LOS } \\
\text { (d) }\end{array}$ \\
\hline Continuous variables & $P$ value & $P$ value & $P$ value \\
\hline Weight at surgery (kg) & .816 & .113 & $(-) .031$ \\
\hline Age at surgery (d) & .294 & .262 & .223 \\
\hline CPB time (min) & .117 & $(+) .033$ & $(+) .041$ \\
\hline CVP $(\mathrm{mm} \mathrm{Hg})$ & $(+) .015$ & $(+) .004$ & $(+) .001$ \\
\hline TP gradient $(\mathrm{mm} \mathrm{Hg})$ & $(+) .011$ & $(+) .049$ & $(+) .002$ \\
\hline $\mathrm{CA}(\mathrm{mm} \mathrm{Hg})$ & .898 & .465 & .807 \\
\hline \multicolumn{4}{|l|}{ Discrete/binary variables } \\
\hline Right ventricular morphology & .793 & $(+) .020^{*}$ & $(+) .011^{*}$ \\
\hline Bilateral superior vena cava & .79 & .581 & .395 \\
\hline Presence of interrupted IVC & .78 & .377 & .578 \\
\hline Previous operations & .655 & .377 & .365 \\
\hline Use of cardiopulmonary bypass & .818 & .783 & .681 \\
\hline Use of cardioplegic arrest & .318 & .410 & .231 \\
\hline Use of fibrillatory arrest & .766 & .166 & .491 \\
\hline Presence of antegrade PA flow & .5 & .995 & .815 \\
\hline Hemi-Fontan construction & .894 & .909 & .924 \\
\hline Concomitant operations & .749 & .358 & .422 \\
\hline
\end{tabular}

$A V$, Arteriovenous. 
TABLE 6. Summary of univariate analysis for any complication

\begin{tabular}{|c|c|c|c|c|c|}
\hline Continuous variables & \multicolumn{2}{|c|}{ No complication ( $\mathrm{n}=198$ ) } & \multicolumn{2}{|c|}{ Complications ( $n=72$ ) } & $P$ value \\
\hline Weight at surgery (kg) & \multicolumn{2}{|c|}{$6.4(4.3-25.1)$} & \multicolumn{2}{|c|}{$5.9(4.2-15.1)$} & .171 \\
\hline Age at surgery (d) & \multicolumn{2}{|c|}{$165(76-4155)$} & \multicolumn{2}{|c|}{$161(80-1736)$} & .829 \\
\hline CPB time (min) & \multicolumn{2}{|c|}{$70 \pm 33$} & \multicolumn{2}{|c|}{$85 \pm 35$} & $.002^{*}$ \\
\hline CVP $(\mathrm{mm} \mathrm{Hg})$ & \multicolumn{2}{|c|}{$13.4 \pm 2.5$} & \multicolumn{2}{|c|}{$14.3 \pm 2.8$} & .029 \\
\hline TP gradient $(\mathrm{mm} \mathrm{Hg})$ & \multicolumn{2}{|c|}{$7.7 \pm 2.0$} & \multicolumn{2}{|c|}{$8.3 \pm 2.5$} & .070 \\
\hline $\mathrm{CA}(\mathrm{mm} \mathrm{Hg})$ & \multicolumn{2}{|c|}{$5.8 \pm 2.2$} & \multicolumn{2}{|c|}{$5.8 \pm 2.1$} & .803 \\
\hline Discrete/binary variables & $\mathbf{n}$ & $(\%)$ & $\mathbf{n}$ & $(\%)$ & $P$ value \\
\hline Right ventricular morphology & 82 & $(41 \%)$ & 38 & $(53 \%)$ & .098 \\
\hline Bilateral superior vena cava & 33 & $(17 \%)$ & 11 & $(15 \%)$ & .785 \\
\hline Presence of interrupted IVC & 7 & $(4 \%)$ & 5 & $(7 \%)$ & .238 \\
\hline Previous operations & 161 & $(81 \%)$ & 61 & $(85 \%)$ & .518 \\
\hline Use of cardiopulmonary bypass & 197 & $(99 \%)$ & 72 & $(100 \%)$ & .988 \\
\hline Use of cardioplegic arrest & 60 & $(31 \%)$ & 31 & $(43 \%)$ & .051 \\
\hline Use of fibrillatory arrest & 23 & $(12 \%)$ & 5 & $(7 \%)$ & .271 \\
\hline Presence of antegrade PA flow & 39 & $(20 \%)$ & 13 & $(18 \%)$ & .762 \\
\hline Hemi-Fontan construction & 10 & $(5 \%)$ & 4 & $(6 \%)$ & .869 \\
\hline Concomitant operations & 119 & $(60 \%)$ & 46 & $(64 \%)$ & .604 \\
\hline
\end{tabular}

$C P B$, Cardiopulmonary bypass; $C V P$, central venous pressure; $T P$, transpulmonary; $C A$, commonatrial; $I V C$, inferior vena cava; $P A$, pulmonary artery. ${ }^{*}$ Maintained significance in the multivariate analysis.

chest tube drainage was affected by an elevated central venous pressure $(P=.015)$ and elevated transpulmonary gradient $(P=.011)$.

In the bilateral group, 1 operation was performed without CPB. Otherwise, single superior vena cava cannulation was performed in 19 patients and bilateral superior vena cava cannulation was achieved in 24 patients. By looking specifically at neurologic complications, there was no relationship to single superior vena cava cannulation in the bilateral group $(P=$ .982).

\section{Effects of a Persistent Left Superior Vena Cava}

Weight (median $6.8 \mathrm{~kg}$ vs $6.2 \mathrm{~kg}, P=.038$ ) and age (median 186 days vs 159 days, $P=.001$ ) at the time of surgery were

TABLE 7. Summary of specific analyses

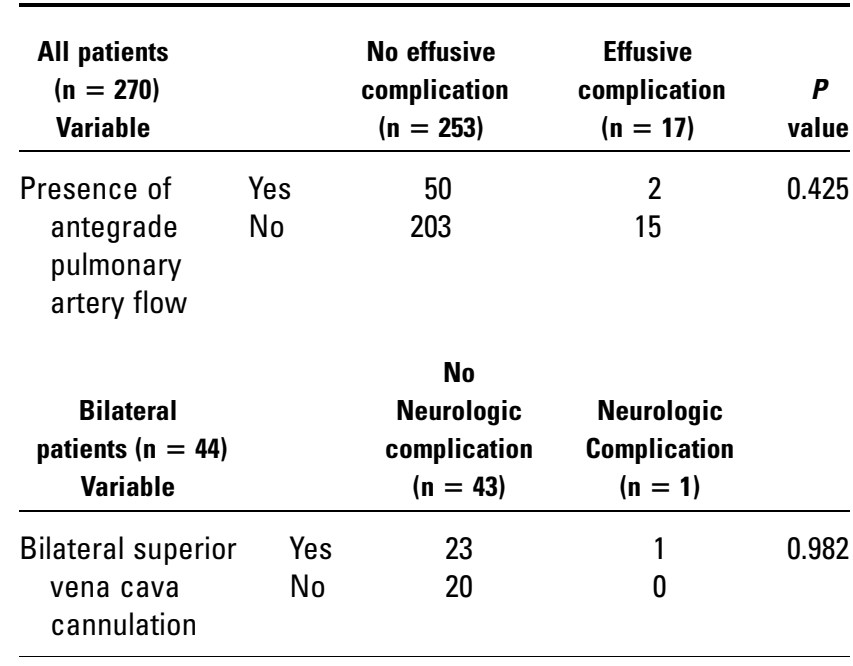

significantly greater in the bilateral bidirectional Glenn group compared with the unilateral group (Table 8). Other significant differences between group characteristics were a higher incidence of an interrupted inferior vena cava (16\% vs $2 \%$, $P=.003)$ and a longer CPB time (105 minutes vs 68 minutes, $P=.0001)$ in the bilateral group. However, there was no difference in any of the outcome variables.

\section{Discussion}

Many children with congenital heart disease require palliation with a bidirectional Glenn procedure. Although some patients with a balanced circulation do not require intervention before the cavopulmonary anastomosis, the majority undergo

TABLE 8. Significant differences between unilateral and bilateral patient groups

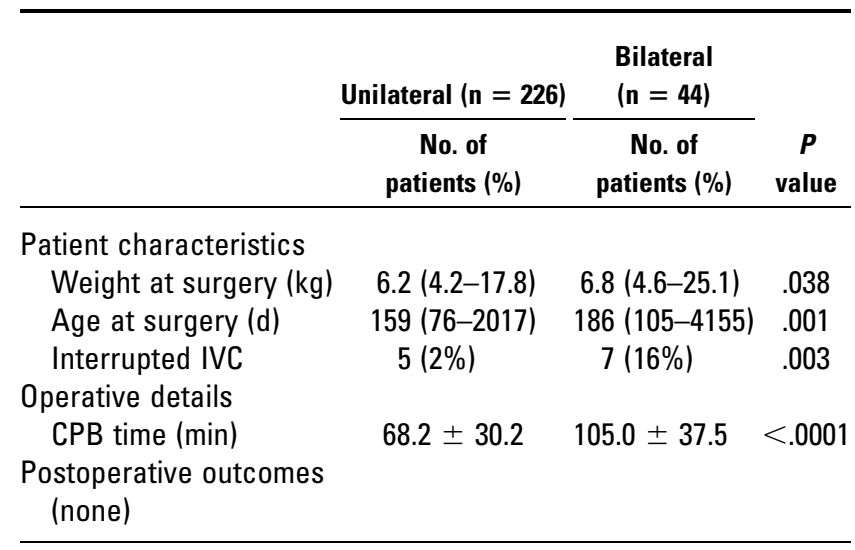

$I V C$, Inferior vena cava; $C P B$, cardiopulmonary bypass. 
prior procedures during early infancy. ${ }^{1}$ These typically include a modified Blalock-Taussig shunt for diminutive right-sided structures, a Norwood operation for diminutive left-sided structures, and occasionally a pulmonary artery band for pulmonary overcirculation. The presence of a persistent left superior vena cava requires the creation of bilateral bidirectional superior cavopulmonary anastomoses.

\section{Timing of Surgery}

The bidirectional Glenn operation is typically performed before 6 months of age., ${ }^{2,3}$ There are data to show that younger patients, aged less than 4 months, have a longer duration of mechanical ventilation, pleural drainage, intensive care unit stay, and hospitalization. ${ }^{4}$ There are also data to show that very young age, less than 2 months, is a risk factor for increased mortality. ${ }^{3}$ In our series, there was no association between patient age at the time of surgery and duration of pleural drainage, length of intensive care unit stay, length of hospital stay, morbidity, or surgical mortality. On the other hand, lower weight at the time of surgery was associated with prolonged length of hospital stay in our series.

There was a significantly higher age and weight for the patients in the bilateral group compared with the unilateral group. This result was expected, as we attempted to delay the bidirectional Glenn procedure in the presence of a persistent left superior vena cava to facilitate bilateral superior vena cava cannulation and cavopulmonary anastomoses. Although there is not a direct causal relationship, this strategy resulted in equivalent outcomes between the unilateral and bilateral groups. It remains unclear whether the outcomes would be similar if the operations were performed at similar ages and weights to the unilateral group.

\section{Cardiopulmonary Bypass Strategy}

In the absence of concomitant intracardiac procedures, the bidirectional Glenn can be performed without the use of CPB if an existing source of pulmonary blood flow can be maintained during the cavopulmonary anastomosis. ${ }^{2,5}$ This has the advantage of avoiding full heparinization and the risks of CPB. Unfortunately, it has the disadvantage of subjecting the upper body and brain to high venous pressure, which in turn can lead to a low transcranial pressure gradient and neurologic damage. ${ }^{6}$ Although this does not typically result in gross neurologic deficits, subclinical changes do occur within the brain. ${ }^{7}$ These changes are absent or minimal when clamping of the superior vena cava is performed with $\mathrm{CPB} .{ }^{7}$ When performing these operations without $\mathrm{CPB}$, some series have used a shunt to decompress the superior vena cava into the right atrium, although the effectiveness of this technique is controversial. ${ }^{6,7}$ In our series, we chose to use CPB with one exception.

For those procedures performed with $\mathrm{CPB}$, a cannulation strategy must be established. Cannulation of all present superior vena cavae individually and the inferior vena cava/right atrium provides for optimal venous drainage and avoidance of venous hypertension. In our bilateral group, we attempted to cannulate both superior vena cavae, although the benefit was weighed against the possibility of narrowing a small superior vena cava. Although bilateral cannulation was only successful in 57\% of cases, there was no relationship between single superior vena cava cannulation and neurologic complications or overall complications.

\section{Maintenance of Antegrade Pulmonary Artery Flow}

There has also been significant controversy regarding the maintenance of antegrade pulmonary blood flow during the bidirectional cavopulmonary shunt. Advantages of antegrade native pulmonary artery flow include promotion of normal pulmonary artery growth and maintenance of pulmonary artery endothelial function. ${ }^{8-12}$ In addition, humoral factors (hepatic flow) and pulsatile hemodynamics are maintained that may decrease the tendency for aortopulmonary collaterals and pulmonary arteriovenous malformations to form. ${ }^{8,10-13}$ Last, it preserves the ability to catheterize the pulmonary arteries from the femoral veins. Disadvantages of native pulmonary artery flow include excessive pulmonary flow and elevated pressures resulting in persistent pleural effusions and prolonged hospitalization. ${ }^{8,10,14}$ There have also been trends toward decreased survival in patients with residual antegrade pulmonary blood flow. ${ }^{14}$ In our series, antegrade pulmonary artery flow was maintained in $19 \%$ of patients. There was no relationship to duration of chest tube drainage, presence of effusive complications, overall complications, or mortality.

\section{Conclusions}

The results of the bidirectional Glenn procedure as an intermediate stage in the palliation of single ventricle physiology are excellent. Outcomes were adversely affected primarily by prolonged CPB time, elevated central venous pressure and transpulmonary gradient, and right ventricular morphology. Specifically, outcomes were unaffected by the presence of a left superior vena cava, cannulation strategy, or antegrade pulmonary blood flow. There were few differences between the unilateral and bilateral groups, none of which were postoperative outcomes.

\section{References}

1. Reddy V, Liddicoat J, Hanley F. Primary bi-directional cavopulmonary shunt in infants between 1 and 4 months of age. Ann Thorac Surg. 1995; 59:1120-6.

2. Kirklin J, Barratt-Boyes B. Cardiac Surgery. New York, New York: Churchill Livingston, Inc; 1993.

3. Reddy V, McElhinney D, Moore P, Haas G, Hanley F. Outcomes after bi-directional cavopulmonary shunt in infants less than 6 months old. J Am Coll Cardiol. 1997;29:1365-70.

4. Jaquiss R, Ghanayem N, Hoffman G, Fedderly R, Cava J, Mussatto K, et al. Early cavopulmonary anastomosis in very young infants after the Norwood procedure: impact on oxygenation, resource utilization, and mortality. J Thorac Cardiovasc Surg. 2004;127:982-9. 
5. Baue A, Geha A, Hammond G, Laks H, Naunheim K. Glenn's Thoracic and Cardiovascular Surgery. Stanford, Connecticut: Appleton and Lange; 1996.

6. Jahangari M, Keogh B, Shinebourne E, Lincoln C. Should the bi-directional Glenn procedure be performed through a thoracotomy without cardiopulmonary bypass? J Thorac Cardiovasc Surg. 1999;118:367-8.

7. Rodriguez R, Weerasena N, Cornel G. Should the bi-directional Glenn procedure be better performed through the support of cardiopulmonary bypass? J Thorac Cardiovasc Surg. 2000;119:634-5.

8. Xie B, Zhang J, Shetty D. Bidirectional Glenn shunt: 170 cases. Asian Cardiovasc Thorac Ann. 2001;9:196-9.

9. Kurotobi S, Sano T, Kogaki S, Matsushita T, Miwatani T, Takeuchi M, et al. Bidirectional cavopulmonary shunt with right ventricular outflow tract patency: the impact of pulsatility on pulmonary endothelial function. J Thorac Cardiovasc Surg. 2001;121:1161-8.
10. McElhinney D, Marianeschi S, Reddy M. Additional pulmonary blood flow with a bi-directional Glenn anastomosis: does it make a difference? Ann Thorac Surg. 1998;66:668-72.

11. Freedom R, Nykanen D, Benson L. The physiology of the bi-directional cavopulmonary connection. Ann Thorac Surg. 1998;66:664-7.

12. Caspi J, Pettitt T, Ferguson T, Stopa A, Sandhu S. Effects of controlled antegrade pulmonary blood flow on cardiac function after bi-directional cavopulmonary anastomosis. Ann Thorac Surg. 2003;76:1917-21.

13. Frommelt M, Frommelt P, Berger S, Pelech A, Lewis D, Tweddell J, et al. Does an additional source of pulmonary blood flow alter the outcome after a bi-directional cavopulmonary shunt? Circulation. 1995; 92(Suppl 2):240-4.

14. Mainwaring R, Lamberti J, Uzark K, Spicer R. Bi-directional Glenn. Is accessory pulmonary blood flow good or bad? Circulation. 1995; 92(Suppl 2):294-7. 